Transcontinentales

continentales

Sociétés, idéologies, système mondial

$1 \mid 2005$

Réforme et « Grand Moyen-Orient »

\title{
Les idées du monde
}

Jean-Luc Racine

\section{OpenEdition}

Journals

Édition électronique

URL : http://journals.openedition.org/transcontinentales/766

DOI : 10.4000/transcontinentales.766

ISBN : 978-2-8218-1410-3

ISSN : 1775-397X

Éditeur

Editions de la maison des sciences de l'homme

Édition imprimée

Date de publication : 31 décembre 2005

Pagination : 5-17

ISBN : 2200-92168-3

ISSN : 1950-1684

\section{Référence électronique}

Jean-Luc Racine, "Les idées du monde », Transcontinentales [En ligne], 1 | 2005, document 1, mis en

ligne le 10 décembre 2012, consulté le 25 septembre 2020. URL : http://journals.openedition.org/ transcontinentales/766 ; DOI : https://doi.org/10.4000/transcontinentales.766 


\section{Les idées du monde}

Les clés du monde sont de plus en plus complexes. Pour en déchiffrer l'ordre et les désordres, les dynamiques et les blocages, il faut analyser tout à la fois les forces qui structurent le système mondial, celles qui l'altèrent, celles qui le contestent. Il faut décrypter le jeu des sociétés, enracinées dans leur territoire et leur culture, mais aussi ouvertes aux réseaux transnationaux de l'information, des marchés, des migrations, des idéologies. À l'heure où les nations se cherchent, face au jeu de la mondialisation et des constructions régionales, l'histoire est mobilisée, les identités réinventées, l'avenir imaginé. Le jeu inégal des puissances ne verrouille ni les débats, ni les compétitions entre États, ni les aspirations à un monde meilleur. Sont en cause des valeurs, des intérêts, mais aussi des tensions, des crises et des désastres, qui redonnent à l'impératif de développement tout son poids. La quête de sécurité n'est plus seulement militaire : elle doit être aussi économique, sociale, environnementale. La diplomatie doit repenser le droit international. Les concepts de solidarité, de biens publics mondiaux, de sociétés civiles sont entrés dans son vocabulaire. Les ruses de la dialectique sont infinies, comme en témoignent les enchevêtrements du Nord et du Sud, du global et du local, du national et du multilatéral.

Dans ce contexte, il importe d'être à l'écoute du monde, sur les terrains, tout en croisant les analyses et les regards, pour nourrir de façon pertinente les grands débats d'idées. Transcontinentales, comme son nom l'indique, entend travailler dans ce sens. Elle n'est certes pas seule sur ce grand chantier, mais elle dispose d'un outil qui lui est propre, et qui relève d'une exception culturelle bien française : le réseau, transcontinental, des centres et instituts français de recherche à l'étranger. Des centres qui ont une histoire, parfois longue - celui de Damas fut ouvert en 1922 -, parfois récente - Moscou ouvrit en 2001 -, parfois tumultueuse - Kaboul a rouvert en 2003. Des centres qui sont eux-mêmes des têtes de réseau, fécondées par les coopérations nouées avec les chercheurs des pays d'accueil, par les liens maintenus avec ceux qui y furent boursiers ou pensionnaires, et par les opérations conduites avec d'autres institutions de recherche, en France ou à l'étranger.

Implantés dans plus de 25 pays, sur quatre continents, de la Russie à la Chine, de l'Europe au Japon, de l'Inde à l'Asie centrale et à l'Asie du Sud-Est, de la Turquie et de l'Iran au Proche-Orient et au Maghreb, de l'Égypte à l'Afrique du Sud, du Mexique au Pérou, ces centres de recherche français à l'étranger, mobilisant leur expérience, leur savoir et leurs réseaux internationaux, apporteront ici leurs éclairages, leurs constats, leurs hypothèses. Pour aider à mieux comprendre les mutations du monde d'aujourd'hui, la résonance de ses héritages, et ses futurs possibles. 
Soucieuse de croiser les études fines de terrain, les perspectives régionales et les questions contemporaines les plus significatives, Transcontinentales ouvre son premier dossier sur le "Grand Moyen-Orient», une région où le réseau des centres est très implanté.

\section{Réforme et «Grand Moyen-Orient»}

La formule "Grand Moyen-Orient», popularisée par l'administration américaine, est controversée, mais elle s'est trouvée placée au centre de débats d'importance.

\section{L'initiative américaine et ses aléas}

Le 26 février 2003, trois semaines avant le déclenchement de la guerre en Irak, le président américain George W. Bush établit un premier lien entre cette opération et une ambition plus large, celle "d'étendre les valeurs démocratiques» en tirant parti «des signes prometteurs d'un désir de liberté dans le Moyen-Orient», désir manifeste en ce que «des intellectuels arabes ont appelé leurs gouvernements à combler "le déficit de liberté" ». Et Bush d'annoncer qu'un "nouveau régime en Iraq servira de puissant exemple en faveur de la liberté pour les autres nations de la région $»^{1}$. Six mois plus tard, le 6 novembre 2003, le président américain annonce que les États-Unis vont s'engager dans une "stratégie avancée pour la liberté», afin de promouvoir la démocratie dans tout le Moyen-Orient. Un compte-rendu de la MaisonBlanche use de la formule "Grand Moyen-Orient» sans la définir ${ }^{2}$. Le 19 février 2004, on en sait davantage. Un document préparatoire au sommet du G8 prévu pour juin est publié par le quotidien arabe de Londres Al-Hayat : il est intitulé "l'Initiative du Grand Moyen-Orient». Quelques jours plus tard, Le Monde note que "l'expression s'est répandue comme une traînée de poudre» et que cette initiative américaine "est devenue le thème imposé de la diplomatie occidentale», inscrit au prochain sommet européen, au prochain G8 et au prochain sommet de l'OTAN.

L'initiative américaine identifie trois domaines d'action pour favoriser la réforme : promouvoir la démocratie et la bonne gouvernance, développer l'éducation et le savoir, stimuler l'économie par la "libération du potentiel du secteur privé», le microcrédit, le libre-échange, et une Banque régionale de développement. L'action en faveur des femmes est mise en avant dans chacun des trois secteurs. Il s'agit bien, dans l'optique de George W. Bush explicitée le 6 novembre 2003, de tarir les sources du terrorisme par la réforme : «tant que la liberté et la démocratie ne fleuriront pas au Moyen-Orient, cette région demeurera stagnante, pleine de ressentiment et violente - et elle exportera la violence et la terreur vers les nations libres.» Une pluie de critiques assaille aussitôt le projet. Une première objection dénonce un concept qui masque la profonde diversité de la zone. Elle y voit comme un nouvel et dangereux avatar d'un orientalisme primaire simplifiant la réalité pour

1 - President Discusses the Future of Iraq. White House. Office of the Press Secretary. 26 février 2004.

2 - President Bush Calls for a "Forward Strategy of Freedom» to Promote Democracy in the Middle East. The White House. Office of the Press Secretary, 6 novembre 2003. 
mieux définir l'identité supposée de l'Autre. Qu'est donc cet espace étendu sur une vingtaine de pays, de la Mauritanie au Pakistan, sinon un néologisme géographique derrière lequel on n'ose définir explicitement, à la Huntington, une civilisation portée par l'islam? Une seconde critique, un pas plus loin, y voit une erreur stratégique, un risque de cristalliser des blocs - l'Occident, l'islam - au moment même où il convenait, après le 11 septembre, d'éviter les simplifications nourrissant les antagonismes. Un troisième argument met en cause la crédibilité américaine, lançant cette initiative sans consultation préalable avec les pays intéressés, après avoir envahi l'Irak sans mandat de l'ONU, tout en laissant de côté la question israélo-palestienne, alors totalement bloquée.

Washington avait invoqué les deux rapports sur le développement humain dans le monde arabe, publiés en 2002 et en 2003 par une équipe d'analystes et d'universitaires arabes, sous l'égide du Programme des Nations unies pour le développement et du Fonds arabe pour le développement économique et social ${ }^{3}$. Le coordonnateur des rapports, l'Égyptien Nader Fergany, en appelle certes à la réforme, mais endogène. Il dénonce «l'arrogance américaine» et le manque de crédibilité des ÉtatsUnis. Il appelle à rejeter un projet "qui vient de l'extérieur»" Nombre de chefs d'État régionaux, à commencer par les régimes autoritaires traditionnellement amis de Washington, se rebiffent. Hosni Mubarak, le président égyptien, annonce «la violence et le chaos» si ce projet est imposé par Washington. Ahmad Maher, son ministre des Affaires étrangères, assure que l'Égypte "n'attend de consignes de personne pour mener des réformes $»^{5}$. Saud al Faisal, ministre saoudien des Affaires étrangères, rappelle que les Arabes, riches de leur longue histoire, «sont capables de gérer leurs propres affaires $»^{6}$. Un communiqué conjoint égypto-saoudien précise que les deux pays «n'acceptent pas qu'un type particulier de réformes soit imposé de l'extérieur aux pays arabes et musulmans", et rappelle qu'il importe de «trouver des solutions justes» aux causes chères aux Arabes et à la nation islamique, «tout particulièrement aux questions palestinienne et irakienne». L'embarras est tel, que le sommet de la Ligue arabe supposé débattre de la proposition américaine, prévu pour le 29 mars, est annulé in extremis par le pays hôte, la Tunisie. Aux États-Unis même, les critiques de poids ne manquent pas. Zbigniew Brezinski parle de «mauvaise façon de vendre la démocratie au monde arabe», tandis qu'un rapport du Carnegie Endowment for International Peace parle de «faux départ» ${ }^{7}$.

3 - Trois rapports ont été publiés à ce jour. Voir www.undp.org/rbas/adhr. Rapport 2002 sur le développement humain dans le monde arabe. Créer des opportunités pour les générations futures, UNDP, 2003. Rapport 2003 sur le développement humain dans le monde arabe. Construire une société de la connaissance UNDP, 2004 et Rapport 2004 sur le développement humain dans le monde arabe. Vers la liberté dans le monde arabe, UNDP, 2005.

4 - Gilbert Acchar, "Le nouveau masque de la politique américaine au Proche-Orient», Le Monde diplomatique, avril 2004. Acchar cite Nader Fergany, "Critique du projet du "Grand Moyen-Orient" », (en arabe) al-Hayat, 19 février 2004.

5 - Le Monde, 27 février 2004.

6 - Nizar Abdel-Kader, "Promoting Reform Efforts in the Middle East», The Jordan Times, 25-26 juin 2004.

7 - Zbigniew Brzezinski, "The Wrong Way to Sell Democracy to the Arab World », The New York Times, 8 mars 2004. Marina Ottaway \& Thomas Carothers, The Greater Middle East Initiative : Off to a False Start, Carnegie Endowment for International Peace, New York, 29 mars 2004. 
De son côté, l'Union européenne ne ménage pas ses mises en garde. Washington lui a beaucoup emprunté, sauf la méthode. Depuis 1995 le partenariat euroméditerranéen, dit Processus de Barcelone, tente précisément d'avancer dans la voie du dialogue politique, de l'ouverture des sociétés civiles et du développement économique. Des milliards d'euros sont engagés. Le 30 mars 2004, devant le Parlement européen qui débat du Grand Moyen-Orient, Chris Patten, commissaire européen aux relations extérieures, met les points sur les «i», en trois étapes. Il rappelle en premier lieu l'ampleur et les modalités de l'engagement européen. C'est un discours de la méthode : "Notre approche à long terme ne porte des fruits que par étapes, mais travailler sur la base du partenariat, de la consultation et de l'appropriation du processus de réforme par les réformateurs de la région nous donnera des résultats plus solides et plus durables que toutes les tentatives d'imposer la démocratie de l'extérieur, par la persuasion ou par le canon du fusil ». Second point : «si nous voulons sérieusement la réforme du monde arabe, la résolution du conflit arabo-israélien reste une priorité stratégique». Troisième remarque : «j'espère que nos amis d'outre-Atlantique reconnaissent que notre approche fondée sur le long terme et l'émergence du consensus a quelque valeur, fondée qu'elle est sur la construction d'un agenda commun qui respecte les différences d'approche des divers pays. L'initiative américaine du Grand Moyen-Orient a suscité des controverses dans la région, principalement parce que ses objectifs ont apparemment été définis sans réelle consultation dans la région. Dans la fureur ainsi suscitée, peu ont porté attention au contenu. ${ }^{8}$

Au-delà des fermes critiques quant à la forme de la démarche américaine, la convergence des objectifs de fond permet de trouver un compromis, lors du G8 réuni en juin en Floride. Définie comme un «partenariat pour un progrès et un avenir communs», «l'Initiative pour le Moyen-Orient élargi et l'Afrique du Nord» fait enfin place à la nécessité de résoudre le conflit israélo-palestinien, tout en précisant que "les conflits régionaux ne doivent pas être un obstacle aux réformes». Celles-ci ne sauraient être imposées de l'extérieur. La diversité des situations nationales est reconnue, sans qu'elle puisse "servir de prétexte pour empêcher la réforme». Le soutien au gouvernement provisoire irakien "pleinement souverain» est explicite. Pour le reste, les trois axes d'intervention sont maintenus : libertés fondamentales sur le plan politique; éducation généralisée et égalité entre hommes et femmes sur le plan social; appui à l'investissement et aux entreprises sur le plan économique. Des projets plus précis sont désormais définis avec les pays de la région présents au sommet : Turquie, Yémen, Jordanie, Algérie, Bahreïn, Afghanistan et Irak. L'Égypte et l'Arabie saoudite, entre autres, ont boudé l'invitation. Un État d'importance se trouve désormais hors du champ défini par l'Initiative : le Pakistan, puissance nucléaire, État du front dans la lutte contre Al-Qaeda. Ce n'est en rien un ostracisme. À la veille du sommet du G8, le général Musharraf avait précisément lancé sa nouvelle doctrine, en phase avec les impératifs américains : sous le nom de "modération éclairée», le président pakistanais appelait à la fois à la lutte contre le terrorisme, à donner la priorité au développement économique et social par un

8 - http://europe.eu.int/comm/external_relations/news/patten/sp04_165.htm. 
effort interne doublé de soutiens externes, et à la résolution des grandes causes chères aux musulmans, dont celle de la Palestine. Tel qu'il est redéfini, le projet américain laisse toutefois sceptiques bien des analystes bons connaisseurs du monde arabe. À la veille du sommet du G8, l'International Crisis Group publie un rapport très détaillé, depuis Amman, sur la nouvelle initiative de Washington, définie comme «en péril dès la naissance»".

Neuf mois plus tard, commencent à poindre des interrogations moins négatives vis-à-vis du projet réformateur américain. Le Washington Post, le 8 mars 2005, publie une analyse au titre équilibré ${ }^{10}$, qui souligne comment un faisceau d'événements semble tout à coup donner quelque crédit aux conseillers de Bush : élections en Iraq et dans les territoires palestiniens en janvier; signaux jugés positifs en Arabie saoudite (élections municipales) et en Égypte (annonce d'un élection présidentielle à candidats multiples et au suffrage universel), manifestations massives au Liban qui font tomber le gouvernement. On parle de "printemps arabe», et Le Monde ose, sur quatre colonnes à la Une, un constat et une question : «le Proche-Orient bouge : faut-il remercier Bush? $»^{11}$. L'éditorial du même jour est plus sobre. Il prête crédit au président américain d'avoir développé haut et fort «l'idée que les peuples musulmans avaient droit à la liberté, à la démocratie, à la prospérité», tout en parlant "d'acquis fragiles", et en invitant les Européens "à ne pas laisser aux Américains le monopole de la rhétorique démocratique». Au-delà de la région, la «révolution orange» en Ukraine, couronnée par la prise de fonction de Viktor Ioutchenko en janvier 2005, et la «révolution des tulipes» en mars 2005 au Kirghizstan semblent amplifier le mouvement démocratique. Signaux supplémentaires, en mai, le Parlement koweitien donne aux femmes le droit de vote et d'éligibilité à compter de 2007, tandis qu'en juin une femme est pour la première fois ministre de l'émirat. L'hypothèse de l'embellie du premier trimestre 2005 se heurte toutefois assez vite à des réalités qui appellent à plus de réserves. L'enlisement irakien en premier lieu, mais aussi, moins évidents mais tout aussi profonds, les rapports de force qui témoignent, où que l'on porte le regard, des difficultés à faire changer, voire à faire évoluer, les structures socio-politiques.

\section{Regards croisés : terrains et théories de l'ambiguiité}

Le dossier ici présenté, sous le titre "Réforme et Grand Moyen-Orient», n'a pas pour objet de commenter au premier chef la stratégie américaine. Mais celle-ci, hyperpuissance oblige, a dessiné la nouvelle toile de fond devant laquelle est posée de nouveau la grande question de la réforme en pays arabes, et au-delà, en pays musulmans. Mais de quelle réforme parle-t-on? celle de l'islam, qui n'en est certes pas à ses premiers débats? celle des régimes politiques? celle des configurations économiques et sociales? Dans l'entretien qui clôt ce dossier, Olivier Roy souligne

\footnotetext{
9 - International Crisis Group, The Broader Middle East and North Africa Initiative : Imperilled at Birth, Brussels/Amman, 7 juin 2004.

10 - Peter Baker, "Mideast Strides Lift Bush, But Challenges Remain», The Washington Post, 8 mars 2005.

11 - Le Monde, 9 mars 2005.
} 
toute l'ambiguïté du thème de la réforme. Certes, il est des auteurs arabes, au Moyen-Orient et en Occident, qui plaident pour repenser l'islam contre tous les dogmatismes stérilisants ${ }^{12}$. Toutefois, faire d'un aggiornamiento théologique la condition nécessaire de la réforme politique et sociale est «une idée séduisante, mais non fondée», pour l'auteur de L'échec de l'islam politique, qui souligne combien il faut repenser les idées reçues sur les rapports entre culture et religion, et religion et politique. La question clé est autre : comment démocratiser sans légitimité politique? Assassiné le 2 juin 2005 à Beyrouth, Samir Kassir avait publié un an plus tôt ses Considérations sur le malheur arabe, où il invoquait bien plus l'histoire que l'islam pour remonter aux sources du blocage de la nahda, la renaissance culturelle et idéologique marquant aussi bien l'Empire ottoman que le protonationalisme arabe, ce processus de modernisation s'étant heurté, après la Seconde Guerre mondiale, à la double assertion des monarchies conservatrices et des régimes laïcs autoritaires ${ }^{13}$. Il faut aussi rappeler que les émirats du Golfe n'ont pas attendu 2004 pour avancer : les élections ouvertes du Koweit en 1992, la Constitution du Qatar en 1995, celle de Bahrein en 2002 témoignent à la fois d'un mouvement et de ses limites, les familles régnantes gardant la haute main sur le pouvoir.

Cette ambiguïté est au cœur du dossier ici ouvert. On l'a noté, l'embellie du premier semestre 2005 a montré ses limites. Les élections irakiennes n'ont pu régler le différend croissant entre sunnites et chiites, ni a fortiori réduire la stratégie déstabilisatrice de forces terroristes combinant des éléments nationalistes à des épigones d'Al-Qaeda. Au Liban, le grand mouvement populaire, couplé à la pression internationale, a certes abouti au retrait des troupes syriennes, mais les élections du printemps ont montré combien comptaient encore les logiques et les marchandages communautaristes, et quel entregent gardaient les pro-syriens, le Hezbollah entrant au passage, pour la première fois, au gouvernement. On observe donc une double logique à l'œuvre. D'un côté, la fitna, ce que Gilles Kepel a appelé «la guerre au coeur de l'islam $»^{14}$, le jihad armé des ultras ayant semé la division au sein de la communauté des croyants, plus que mobilisé les masses dans l'union. De l'autre, la ligne américaine, dont l'appel à la démocratie est oblitéré par la guerre en Irak et par le blocage du conflit israélo-palestinien, dévaluant ainsi l'aspiration démocratique au bénéfice des régimes autoritaires concédant des réformes superficielles pour mieux consolider leur pouvoir dans des temps incertains, ou au bénéfice d'organisations islamistes usant de l'outil électoral à des fins propres.

Il importe donc tout à la fois de prendre note de ce grand frémissement baptisé peut-être un peu vite "printemps arabe», tout en s'interrogeant sur ce qui se déploie véritablement, ici et là, sur le terrain, afin d'enrichir le débat sur la réforme, concept ambigu. Dans ce contexte, quatre auteurs offrent ici leurs éclairages. Ils

12 - Voir par exemple : Abdelwahab Meddeb, La maladie de l'islam, Paris, Le Seuil, 2002; Rachid Benzine, Les nouveaux penseurs de l'islam, Paris, Albin Michel, 2004; Mohamed Talbi, Plaidoyer pour un islam moderne, L'Aube, 2005; Mohammad Arkoun, Humanisme et islam : combats et propositions, Paris, Vrin, 2005. Parmi d'autres intellectuels arabes, le théologien saoudien Hassan al Maleky ou l'essayiste égyptien Gamal Banna appellent eux aussi, à des degrés divers, à repenser l'orthodoxie.

13 - Samir Kassir, Considérations sur le malheur arabe, Arles, Actes Sud, 2004.

14 - Gilles Kepel, Fitna, guerre au cœur de l'islam, Paris, Gallimard, 2004. 
montrent tous, sous des formes diverses, combien le structurel reste présent, et aussi comment le mouvement lancé par l'administration américaine, tout en plaçant l'idée démocratique au cœur de débat, peut ouvrir ou laisser des opportunités à des forces établies comme à des mouvements contestataires qui n'ont assurément pas pour modèle la société démocratique imaginée par la rhétorique occidentale. Mais après tout, il y a plus de dix ans que Ghassan Salamé a dépeint ces « Démocraties sans démocrates $»^{15}$... Il ne faut voir dans ce constat aucune trace d'une quelconque inadéquation entre démocratie et Moyen-Orient. On observe bien plutôt la marque d'une phase historique de recherche de voies nouvelles, balançant entre l'échec déjà ancien du nationalisme arabe, l'échec plus récent de l'islam politique porté par l'inspiration salafiste, et la quête d'une sortie de crise aux paramètres à la fois géopolitiques et socio-économiques.

\section{L'Arabie saoudite}

Le premier dossier de Transcontinentales s'ouvre par une plongée au cœur du monde arabe, à Riyad, dans les quartiers de la capitale saoudienne, alors que s'y jouent, début 2005, des élections municipales - les premières depuis plus de quarante ans. Pascal Ménoret y décrit comment les militants de la Sahwa islamiyya ont habilement subverti un code électoral promouvant l'apolitisme, pour mener un combat à la fois contre le pouvoir et contre les laïcistes, perçus comme la cinquième colonne de l'Occident impérialiste et libéral, les élections fournissant «un moyen de réinvestir la formidable énergie sociale et politique dégagée par l'activisme islamique». L'exercice électoral saoudien fut certes mesuré : $15 \%$ d'inscrits, tous masculins, et parmi eux, seulement $11 \%$ de votants. L'enjeu l'était tout autant : il ne s'agissait que d'élire une partie des conseils municipaux sans pouvoir de décision. Mais cette élection en demiteinte a permis à l'auteur de tirer deux conclusions. D'une part, «le mouvement islamique ne devient pas "post-islamiste" de se prêter, lorsque les autres portes sont fermées, à l'épreuve des urnes. Il ne se dissout pas non plus dans la "social-démocratie", mais conserve toute sa force contestatrice, tant le capital militant est susceptible d'être converti, selon les circonstances et l'évolution des rapports de domination politique, en paisibles dispositions électorales ou en violente opposition armée». D'autre part, «la réticence à se couler dans la rhétorique universaliste d'un Occident perçu comme unilatéral et belliqueux ne peut en aucun cas être interprétée comme un refus de l'universel». Pour Ménoret, la Sahwa islamizya ne cherche pas à refuser ou à accepter la démocratie. Il s'agit pour elle, "d'abord et avant tout, de s'opposer par tous les moyens, démocratiques ou non, à un système de gouvernement jugé injuste et violent». Et l'auteur d'en tirer un axiome : «un pouvoir violent produit une opposition violente, tandis qu'un pouvoir démocratique engendre une opposition pacifique». On le voit, sous les tentes des candidats en campagne électorale, de grandes questions transcendent, sans les nier, les spécificités du contexte local.

\section{Théories de la réforme}

La modestie des réformes entreprises par les régimes saoudien et égyptien, et avant eux par les émirats du Golfe, est-elle le signe annonciateur de changements de fond 
beaucoup plus substantiels? Jean-Noël Ferrié, fort de travaux conduits aussi bien au Maghreb qu'au Moyen-Orient, prend une position tranchée à cet égard. Sur fond de changement de stratégie américaine, qui considère désormais que «la stabilité des gouvernants ne préjuge pas de la fiabilité des peuples» et qui prône tout à la fois «le volontarisme en Irak et le gradualisme en Égypte», Jean-Noël Ferrié s'attache à monter les limites du réformisme gradué, qui sont généralement «des stratégies de survie et des aménagements de l'autoritarisme» relevant de «la simple libéralisation politique». Mais l'autre option, celle d'une transition accélérée résultant d'un «événement critique», comme le furent la transformation puis l'effondrement de l'URSS, ne nourrit pas pour autant une théorie nécessairement plus efficace de la réforme. D'un côté, le gradualisme prédit davantage la sortie de l'autoritarisme qu'il ne nous dit «comment elle se fait». De l'autre, la théorie de la transition part d'une crise de régime, mais ne "dit pas comment on en arrive là». Pour Ferrié, au total, «ce qui fait la différence entre un signal déclenchant un événement critique et un signal déclenchant une simple politique de libéralisation tient en trois points : d'abord le contexte d'anticipation des conséquences de ce signal; puis l'apparition et l'élargissement croissant d'une fracture entre les performances des gouvernants et les attentes de la population; enfin l'affaiblissement subséquent de la crédibilité du statu quo favorable au régime, de sorte qu'il suffit d'un signal pour que les préférences déjà formées s'expriment». À cette aune, conclut l'auteur, "l'élargissement croissant d'une fracture entre les performances des gouvernants et les attentes de la population, qui fut à l'origine [...] de l'effondrement des régimes à l'Est, n'est tout simplement pas à l'ordre du jour dans le monde arabe, quoiqu'il y paraisse». D'abord, parce que les régimes arabes affichent des ambitions réformatrices bien moindres. Ensuite parce que les politiques gradualistes «viennent apporter aux régimes les ressources nécessaires à la gestion de ce différentiel ", par le double biais de réformes politiques minimales et d'aides au développement.

\section{Les camps palestiniens du Liban}

Peut-on décrypter les évènements libanais à la lumière de ces réflexions? Bernard Rougier rappelle d'abord comment la dynamique du changement, lancée par les manifestations populaires consécutives à l'assassinat de Rafic Hariri, «s'est heurtée à la réalité du système confessionnel libanais et de ses pièges, ainsi qu'à la résistance des alliés politiques de la Syrie», qui ont réussi «à empêcher la manifestation du 14 mars 2005 de se transformer en un mouvement national transcommunautaire». Il éclaire ensuite comment Damas, poussé par la communauté internationale à évacuer le Liban au terme de la résolution 1559 de l'ONU, a usé des services de sécurité syro-libanais pour maintenir son influence sur les camps palestiniens du Liban, bastion décisif du Hezbollah qui refuse de désarmer tout en entrant au gouvernement. Fin connaisseur du très complexe échiquier des camps, Bernard Rougier y montre des Palestiniens divisés, et le poids des factions opposées à Yasser Arafat, bête noire de Damas, puis à son successeur Mahmoud 'Abbas. Aiguisés par la situation internationale, les prêches des idéologues jihadistes des camps visent à «détruire la croyance en la légitimité des institutions palestiniennes». Ainsi, «le discours de solidarité avec l'environnement islamique et arabe éloigne les acteurs palestiniens du système international, alors que celui-ci apparaît comme le seul moyen pour eux d'éviter le face à face inégal avec un État aussi puissant qu'Israël ». 
La «révolution palestinienne» perd ainsi de son lustre et de son épaisseur historique, «en étant amalgamée à d'autres luttes, en Tchétchénie, en Afghanistan ou en Irak». Après les élections de 2005, la nouvelle équipe dirigeant le Liban, «formée autour du clan Hariri, n'a pas cherché à briser le monopole construit par la Syrie et ses alliés sur le dossier palestinien au Liban, ni voulu entraver la diplomatie déployée par le Hezbollah dans le milieu des camps». Damas et ses alliés, usant du refus libanais d'intégration définitive des réfugiés dans le pays, réussissent donc "à empêcher le gouvernement de définir une politique alternative».

Derrière les grands mouvements de foule du printemps 2005, et le retrait officiel des forces syriennes, le Liban ne semble donc pas changer tant que cela. Pourtant, une nouvelle dynamique est à l'œuvre. Elle est lancée par la commission d'enquête internationale sur l'assassinat de Rafic Hariri, qui fait part de ses premiers résultats devant le Conseil de sécurité des Nations unies, fin octobre. L'enquête déstabilise les services de sécurité syro-libanais, et la résolution 1636, présentée par la France et par les États-Unis, et adoptée à l'unanimité, intensifie fortement la pression sur le président syrien Bachar el Assad et sur son obligé, le président libanais Émile Lahoud. Sans préjuger de l'avenir, le processus ici retenu - coopération franco-américaine, et unanimité négociée au Conseil de sécurité contraste fortement avec celui qui a mené à la guerre en Iraq. L'usage de la force n'est envisagé, en demi-teinte, que par l'inscription de la résolution 1636 au chapitre viI de la charte des Nations unies, qui l'autorise sous certaines circonstances. Le vrai débat entre puissances porte en fait sur l'usage, ou non, de sanctions contre Damas. L'épisode illustre en tout cas les limites mais aussi les effets des mobilisations populaires, le poids de la diplomatie internationale, sa prudence, et la complexe dialectique entre États et sociétés.

\section{L'Asie centrale}

Hors du «Grand Moyen-Orient» défini par l'administration américaine et par le G8, l'Asie centrale trouve tout naturellement sa place dans ce dossier puisque, audelà d'une continuité géographique et culturelle évidente avec ses voisins du Moyen-Orient élargi, la question de l'évolution des régimes autoritaires en sociétés musulmanes s'y pose aussi. Mais les spécificités régionales sont fortes, l'héritage soviétique ayant laissé sa marque sur les structures du pouvoir. Après l'effondrement de l'URSS en 1991, les nouvelles républiques d'Asie centrale ont redéfini les rapports de l'État à l'islam, avec un objectif simple : accorder une légitimité accrue aux milieux religieux pour mieux les contrôler. Sébastien Peyrouse éclaire ici les relations entre pouvoirs d'État et question religieuse, "clé privilégiée pour réfléchir à la réforme et à ses enjeux dans des pays de tradition musulmane officiellement laïcs». Dans un système politiquement verrouillé, l'État autoritaire fait de facto reposer sur «l'islam officiel » qu'il contrôle le rôle de régulateur des aspirations populaires, afin d'écarter à la fois la menace de l'islamisme radical et celle d'un mouvement démocratique. La «révolution des tulipes» au Kirghizstan en mars 2005, et les manifestations d'Andijan durement réprimées par le pouvoir ouzbek deux mois plus tard, témoignent de l'usure de ce modèle post-soviétique, mais ne marquent pas pour autant la victoire du contre-modèle américain. L'Asie centrale s'agite en 2005, mais sans reprendre les scénarios géorgien et ukrainien. 
En Ouzbékistan, Islam Karimov reste aux commandes, tout en voyant sa position internationale affaiblie. Au Kirghiztan, le soulèvement populaire a certes mis un terme à quinze ans de régime d'Askar Akaev, mais les élections présidentielles de juillet 2005 et la constitution du nouveau gouvernement traduisent bien moins un grand élan démocratique que l'élaboration d'un compromis entre piliers de l'ancien pouvoir, le président Kourmanbak Bakiev, ancien premier ministre d'Akaev, ayant choisi comme chef du gouvernement son concurrent Felix Koulov, ancien chef des services secrets.

Moscou a appuyé dans l'ombre ce compromis entre clans du Sud et du Nord du pays, et pour l'heure, tire les marrons du feu. Dénonçant une stratégie américaine derrière les troubles récents dans la région, le président ouzbek a signé en juin à Moscou un pacte de défense qui renforce l'influence russe dans le pays clé de l'Asie centrale, qui s'était ouvert plus que d'autres aux sirènes occidentales. Comme Bakiev, Karimov a également demandé à Washington de définir un calendrier d'évacuation des bases U.S. ouvertes dans la région au nom des opérations lancées contre l'Afghanistan des Talibans après le 11 septembre. Le 2 juillet, au sommet russo-chinois de Moscou, Vladimir Poutine et Hu Jintao avaient rejeté les tentatives d'imposer sur des États souverains des «modèles de systèmes socio-politiques étrangers ». Trois jours plus tard, au sommet de l'Organisation de coopération de Shanghai, russes, chinois et pays d'Asie centrale dénonçaient de concert «les tentatives de monopoles et de domination sur les affaires internationales» et rappelaient, comme en écho aux commentaires entendus en haut lieu en Égypte ou en Arabie saoudite quelques mois plus tôt, que «les modèles concrets de développement social ne pouvaient être exportés». Sébastien Peyrouse le rappelle en conclusion : «les changements proclamés en matière religieuse restent pour les régimes un moyen de mettre en avant leur volonté de réforme», mais ce discours officiel éculé ne saurait cacher l'autoritarisme post-soviétique particulièrement répressif en Ouzbékistan et en Turkménistan. Par la même, ces dictatures, faute d'ouverture à la contestation démocratique, "accélèrent l'ancrage social d'un islam alternatif, que celui-ci soit appelé à se radicaliser ou non». Dans une région riche en ressources énergétiques et minières, les grands voisins, russe et chinois, n'entendent ni laisser croître l'influence américaine, ni laisser l'islam radical se développer. Ce sont eux qui jouent, comme Washington jadis au Moyen-Orient, la carte de la stabilité, tandis que l'évolution kirghize montre que le travail américain sur les sociétés civiles ne joue pas toujours comme il l'a fait en Ukraine. La théorie de la transition ne manque pas de sujets d'analyse...

\section{Variations}

Un autre lot d'études, hors dossier, le complète à deux égards, par des apports directs aux problèmes de la réforme et de la transition, ou pour rappeler qu'audelà du Moyen-Orient élargi, l'hyperpuissance américaine doit aussi compter avec des pays émergents.

Un premier article éclaire combien, à la base, il est difficile de mettre en place des réformes au quotidien. Nous sommes au Madhya Pradesh, un grand État de l'Inde 
centrale, et depuis une dizaine d'années le gouvernement tente de pallier les lacunes de l'enseignement public primaire, en mettant en ouvre un «programme de garantie de l'éducation", qui vise à créer en milieux défavorisés des établissements à moindre coût, dont les enseignants, formés sommairement, viennent des villages où le réseau de ces écoles alternatives s'implante. François Leclercq dresse au terme d'une enquête fine le bilan de cette expérience. Certes, l'augmentation des taux de scolarisation est nette, mais la qualité de l'enseignement, faute de moyens, est faible. Plus dépendante des conditions locales que les établissements publics standards, cette extension du système scolaire «tend ainsi à refléter la société rurale plutôt qu'à la transformer». La hiérarchie scolaire renvoie à la hiérarchie sociale : «les enfants tribaux et de basse caste ont pour l'essentiel accès aux écoles du programme de garantie de l'éducation; les enfants des castes intermédiaires aux autres écoles publiques et les enfants de hautes castes aux écoles privées». Si l'extension de la scolarisation est en soi une avancée, elle reflète aussi, dans ses modalités inégales, un choix politique.

Le second article nous ramène à la question de la transition et du politique, puisqu'il porte sur les riches débats qui ont marqué, en pays tchèques, la mise en place de la démocratie post-communiste. Magdaléna Hadjiisky en souligne l'originalité et les enjeux. Dans l'effervescence de la transition pacifique qui a mis un terme au régime pro-soviétique, le Forum civique de Vaclav Havel entendait promouvoir avant tout la participation citoyenne cherchant à concilier la recherche du consensus et les expressions minoritaires, au nom d'une "politique non-politique», privilégiant l'homme sur les appareils. Assez vite pourtant, une conception plus classique mit en avant, plutôt que la participation citoyenne, la démocratie délégative, légitimant de nouveau le jeu des partis tout en optant pour le libéralisme économique. Il est remarquable, note Magdaléna Hadjiisky, «que la controverse la plus importante sur la nature du régime en construction soit apparue non pas entre les démocrates et les communistes lors des tables rondes, ni entre les différents partis du camp démocratique, mais à l'intérieur du Forum civique luimême», le fer de lance du mouvement pour la transition. Derrière les spécificités du cas étudié - «l'héritage critiqué de la première République» tchèque, «le souvenir de l'État-Parti » des années communistes, et «l'héritage également débattu du Forum civique»-, les pays tchèques apportent ainsi une expérience unique à la problématique de la construction des régimes post-autoritaires.

Loin de ces questions, le dernier article de ce premier numéro de Transcontinentales s'ouvre vers les larges horizons de la nouvelle Asie. Il éclaire une autre transition, aux enjeux essentiels : celle de la montée en puissance de la Chine et de l'Inde. En portant le regard sur la façon dont ces deux grands pays se positionnent en Asie du Sud-Est pour tirer parti des nouvelles dynamiques de construction régionale, Isabelle Saint-Mézard analyse les architectures en construction autour de l'ASEAN, et le croisement des logiques économiques et des ambitions géopolitiques qui animent une immense région en forte croissance. En même temps, elle décrypte le jeu de miroirs par lesquels la Chine et l'Inde s'observent, et quelles conclusions stratégiques Pékin et New Delhi en tirent. Si l'asymétrie reste de règle entre les deux pays, au bénéfice de la Chine, Pékin n'en prend pas moins l'Inde au sérieux, 
désormais. Une double logique est ainsi à l'œuvre. D'un côté, la Chine "semble soucieuse de s'assurer que l'Inde ne porte pas ombrage à sa prééminence en Asie orientale», tandis que l'Inde «semble peu disposée à cautionner, encore moins à soutenir, les projets régionaux promus par Pékin». Mais en même temps, «L'Inde admet qu'une amélioration des liens avec la Chine aidera, entre autres choses, à renforcer son intégration au régionalisme asiatique», tandis que "du point de vue chinois, la meilleure option pour gérer les ambitions indiennes reste de renforcer le climat de confiance et de coopération, de façon à désamorcer toute friction à venir». Cette ligne n'empêche pas les deux voisins d'investir fortement dans la défense, tout en développant leurs relations économiques. On a vu plus haut Russie et Chine jouer leurs pions en Asie centrale, face à l'activisme américain. On observe ici, sur les façades maritimes de l'Asie, Inde et Chine prendre leurs marques. Moscou, Pékin et New Delhi n'en cherchent pas moins de bonnes relations avec Washington, source de capitaux et de technologies avancées. Le temps des blocs est révolu, celui du Grand Jeu, moins ouvertement belliqueux que jadis, change d'échelle, quelle que soit, en ce début de xxi siècle, l'étendue de la suprématie américaine.

\section{Panorama}

La rubrique des comptes rendus joue également sur la dualité qui marque les articles de ce premier numéro de Transcontinentales. On y trouvera tour à tour l'écho de publications qui apportent elles aussi leur contribution aux problématiques abordées dans le dossier, et celui de travaux éclairant, dans l'épaisseur de l'histoire comme dans les déploiements contemporains, d'autres questionnements. En ouverture, l'écho d'un dialogue franco-maghrébin sur les «savoirs du lointain" interroge les concepts et les pratiques des sciences sociales dans les espaces qu'on appelle parfois, faute de mieux, "aires culturelles», au risque de sous-estimer aujourd'hui les mutations qu'elles subissent sous le choc de la mondialisation, mais aussi leurs contributions propres à cette mondialisation. Ce jeu d'échelles entre local, régional et global; ce va-et-vient entre Nord et Sud ou de Sud à Sud; cette interrogation sur les risques symétriques que portent d'une part les clichés culturalistes, qui enferment l'autre dans des essentialismes identitaires aux rigidités imaginaires, et d'autre part les idéologies politiques ou les modèles économiques qui sous-estiment la réalité et résilience des ancrages qui définissent peuples, nations et civilisations : voilà autant de questions que Transcontinentales devrait croiser en permanence. La difficulté majeure est sans doute là : ne pas gommer le spécifique, ni le surévaluer, pour mieux définir les articulations entre diversité culturelle et universalité. En complément au dossier, sont également analysés des ouvrages sur la genèse ambiguë de la modernité intellectuelle égyptienne, ou sur le rôle, débattu, des organisations non gouvernementales comme instruments de la réforme dans le monde arabe et soudanais. D'autres comptes rendus commentent des recherches conduites de par le monde, sur la violence urbaine en Afrique, les soubresauts de la révolution culturelle en Chine, la place des femmes dans la vie politique en Asie du Sud, et ou le dialogue noué de longue date entre le Mexique et la France. 
Un aperçu du sommaire de quelques-unes des revues publiées par les centres, au Maroc, au Yémen, en Syrie, à Prague, à Tokyo, à Hong Kong et à Mexico, ajoute par touches emblématiques à ce vaste panorama, première livraison d'un savoir toujours inachevé, mais qui fait sens.

Cette quête de sens a animé ceux qui, au sein du ministère des Affaires étrangères, ont voulu que naisse une nouvelle revue contribuant à diffuser les analyses et les questionnements d'un réseau à la recherche des idées du monde : en premier lieu Claude Blanchemaison et Gilles Andréani, et leurs successeurs à la Direction générale de la coopération et du développement et au Centre d'analyse et de prévision, Philippe Étienne et Pierre Lévy. Rien n'aurait été possible sans leur appui, sans l'intérêt d'Antoine Grassin, directeur de la coopération scientifique et universitaire, et sans l'engagement décisif de Philippe Georgeais, responsable des sciences sociales et de l'archéologie, puis de Michel Pierre, son successeur. Leurs collaborateurs, Pascal Lemaire au premier chef, ont aidé à faire naître ce premier numéro.

L'appui de la fondation Maison des sciences de l'homme a été également déterminant. Ses administrateurs successifs, Maurice Aymard puis Alain d'Iribarne, ont activement soutenu ce projet, et le responsable des Éditions MSH, Jean-Michel Henny, a toujours été présent quand son expérience était requise. Au CNRS, Gérard Lenclud, directeur du département des sciences de l'homme et de la société, a encouragé cette initiative, à l'heure où le ministère des Affaires étrangères et le CNRS travaillent en commun à la transformation de certains des centres en unités mixtes de recherche. Chez Armand Colin, le soutien accordé dès le premier jour par la direction éditoriale des revues universitaires où se sont succédé Jean-Louis Soubret et Nathalie Larmanjat, a permis à Transcontinentales de prendre forme.

Quant au fond, il résulte de la confiance accordée d'emblée à une revue encore inexistante par les auteurs et leurs évaluateurs, par les rédacteurs de comptes rendus et par tous ceux qui ont joué un rôle discret mais efficace d'intermédiaires et de stimulateurs. Parmi ceux-ci, il faut d'abord évoquer celles et ceux qui ont bien voulu rejoindre le comité de rédaction - beaucoup d'entre eux ont dirigé des centres -, et les personnalités françaises et étrangères qui, en siégeant au conseil scientifique international de Transcontinentales, lui prêtent une part de leur prestige et de leur expérience. Last but not least, Évelyne Séguy et Marion Gentilhomme ont elles aussi fait le pari de cette aventure, en mettant leurs compétences et leur efficacité à son service. Que toutes et tous en soient remerciés.

Jean-Luc Racine 
